\title{
Magnus and Iordanskii Forces in Superfluids
}

\author{
C. Wexler \\ Department of Physics, Box 351560, University of Washington, Seattle, WA 98195-1560
}

(April 1997)

\begin{abstract}
The total transverse force acting on a quantized vortex in a superfluid is a problem that has eluded a complete understanding for more than three decades. In this letter I propose a remarkably simple argument, somewhat reminiscent of Laughlin's beautiful argument for the quantization of conductance in the quantum Hall effect [1], to define the superfluid velocity part of the transverse force. This term is found to be $-\rho_{s} \boldsymbol{\kappa}_{s} \times \boldsymbol{v}_{s}$. Although this result does not seem to be overly controversial, this thermodynamic argument based only on macroscopic properties of the superfluid does offer a robust derivation. A recent publication by Thouless, Ao and Niu [2] has demonstrated that the vortex velocity part of the transverse force in a homogeneous neutral superfluid is given by the usual form $\rho_{s} \boldsymbol{\kappa}_{s} \times \boldsymbol{v}_{V}$ [3]. A combination of these two independent results and the required Galilean invariance yields that there cannot be any transverse force proportional to the normal fluid velocity, in apparent conflict with Iordanskii's theory of the transverse force due to phonon scattering by the vortex [4].
\end{abstract}

Typeset using REVTEX 


\section{INTRODUCTION}

The importance of quantized vortices in superfluids has been recognized since Onsager first put forth the idea of quantization of circulation almost 50 years ago [5.6]. A vortex moving in a superfluid experiences a force transverse to its velocity which is equivalent to the Magnus or Kutta-Joukowski hydrodynamic lift present in classical hydrodynamics [7], which is generally written [3]

$$
\boldsymbol{F}=\rho \boldsymbol{\kappa} \times\left(\boldsymbol{v}_{V}-\boldsymbol{v}_{\text {fluid }}\right) .
$$

However, there is no consensus on the problem of generalizing the Magnus force to the superfluid case, and various inequivalent expressions for the relevant forces abound [2,8 8 , 13].

This letter deals specifically with the calculation of the superfluid velocity part of the Magnus force, that is the transverse force that depends on the superfluid velocity $\boldsymbol{v}_{s}$. It is shown that this force is given by

$$
\boldsymbol{F}_{s}=-\rho_{s} \boldsymbol{\kappa}_{s} \times \boldsymbol{v}_{s} .
$$

Recently Thouless, Ao and Niu [2] (TAN) have convincingly argued for a universal vortex velocity part of the Magnus force given by

$$
\boldsymbol{F}_{V}=\rho_{s} \boldsymbol{\kappa}_{s} \times \boldsymbol{v}_{V}
$$

for uniform neutral superfluids; and Geller, Wexler and Thouless have generalized TAN's results to charged systems in the presence of a periodic potential [14]. The natural assumption by TAN that the normal fluid does not circulate around the vortex $\left(\kappa_{n}=\oint \boldsymbol{v}_{n} \cdot d \boldsymbol{l}=0\right)$ has been confirmed by a recent calculation [13] in the thermodynamic limit (mean free path of excitations much smaller than system size). There is an important point to notice: TAN deals only with the part of the transverse force that depends on the vortex velocity, while no statement is made regarding the other parts of this force that depend on the normal and superfluid velocities.

The confusion in the topic of writing the various parts of the Magnus force is widespread. Part of it arises from different interpretations on the role played by excitations, and whether or not these are scattered asymmetrically by the vortex [4] leading to a transverse force (the Iordanskii force) proportional to the normal fluid density $\rho_{n}$ and either the relative velocity $\left(\boldsymbol{v}_{n}-\boldsymbol{v}_{V}\right)$ or $\left(\boldsymbol{v}_{n}-\boldsymbol{v}_{s}\right)$.

The results presented in this letter, combined with TAN and Galilean invariance, are incompatible with the existence of a transverse force proportional to the normal fluid velocity $\boldsymbol{v}_{n}$.

I must note that this letter does not deal with the determination of additional dissipative terms (namely the longitudinal forces), which are negligible under the conditions of the present work. This is an interesting subject as well, but the arguments presented only determine the transverse forces.

In sec. II I write down the most general transverse force, linear in the velocities, that is compatible with Galilean invariance. Section III is the main section of the letter, where the the superfluid velocity part of the Magnus force is calculated. In sec. IV I write the final form of the transverse force by combining the results of this letter and TAN, plus Galilean invariance. I also discuss the diverse results obtained by other authors and their assumptions. 


\section{GALILEAN INVARIANT TRANSVERSE FORCE}

In a homogeneous superfluid the forces acting on a vortex must be expressed in terms of velocity differences only. Consider a vortex moving with velocity $\boldsymbol{v}_{V}$ in a superfluid where the superfluid component has an asymptotic velocity $\boldsymbol{v}_{s}$ and the normal component $\boldsymbol{v}_{n}$. The most general Galilean invariant transverse force can be written as

$$
\boldsymbol{F}=A \hat{\boldsymbol{\kappa}} \times\left(\boldsymbol{v}_{V}-\boldsymbol{v}_{s}\right)+B \hat{\boldsymbol{\kappa}} \times\left(\boldsymbol{v}_{V}-\boldsymbol{v}_{n}\right),
$$

where $A$ and $B$ are constants to be determined and $\hat{\boldsymbol{\kappa}}$ is a unit vector pointing in the direction of the vortex line. Our task is the determination of these unknown coefficients. It is customary to divide this expression into separate terms, each involving one particular velocity and denote them accordingly: the vortex velocity part of the Magnus force $(A+$ $B) \hat{\boldsymbol{\kappa}} \times \boldsymbol{v}_{V}$, the superfluid velocity part $(-A) \hat{\boldsymbol{\kappa}} \times \boldsymbol{v}_{s}$, and the normal fluid velocity part $(-B) \hat{\boldsymbol{\kappa}} \times \boldsymbol{v}_{n}$. Knowledge of two of these forces completely determines the third. In the following section I determine the coefficient $A$ by calculating the superfluid velocity part of the Magnus force.

\section{FREE ENERGY AND FORCE}

Here I wish to present a very simple gedanken experiment, whose outcome will determine the coefficient $A$ as mentioned above. This argument has some parallels to Laughlin's own thought experiment relating to the quantization of Hall conductance in the quantum Hall effect [1].

Consider a neutral superfluid trapped inside a toroid like the one shown in the figure. For simplicity assume the toroid to have a roughly uniform section and that the circumference $L_{x}$ is much bigger than $L_{y}$. This makes the superfluid velocity $v_{s}$ approximately uniform, which is what is actually desired for a definition of the superfluid velocity part of the Magnus force. I must remark that the argument is more general, and these assumption are merely necessary to keep the argument clean and simple.

Assume that in the initial state $N \gg 1$ quanta of circulation are trapped in the toroid so that the superfluid velocity is given by $v_{s}=N h / m L_{x}$. Under this condition the normal fluid is pinned to the container and $v_{n}$ is zero (in fact, this is how the normal density $\rho_{n}$ is normally defined [15]). At some initial time a vortex is created at the outer edge and slowly dragged towards the center of the ring by some means [2], where it is annihilated at a later time $t=\tau \rightarrow \infty$. The final state corresponds to a trapped circulation $(N+1) h / m$, while

the normal fluid will still be at rest. By performing this process very slowly, dissipative effects are negligible.

While transporting the vortex across the ring, one needs to perform work on the system. In terms of eq. (4), and given the fact that $v_{V}=v_{n}=0$, the work is given by integrating the force along the displacement $d \boldsymbol{r}$ of each vortex segment $d \boldsymbol{l}$ :

$$
\begin{gathered}
W=-\int A\left(d \boldsymbol{l} \times \boldsymbol{v}_{s}\right) \cdot d \boldsymbol{r}=A \int(d \boldsymbol{l} \times d \boldsymbol{r}) \cdot \boldsymbol{v}_{s} \\
=A \int d \boldsymbol{S} \cdot \boldsymbol{v}_{s}=A\left(L_{y} L_{z}\right) v_{s} .
\end{gathered}
$$


For isolated systems the change in energy corresponds to the amount of work. The argument is straightforward but it is much simpler to consider an isothermal process. The amount of work performed then corresponds to the variation of the Helmholtz free energy $\mathcal{A}=\mathcal{E}-T S$ [16]. The free energy can be expressed in terms of the energy of the ground state plus the free energy of excitations:

$$
\mathcal{A}=E_{\text {g.s. }}+\mathcal{A}_{\text {excit }}
$$

One will be interested in the variation of the free energy due to a variation of the superfluid velocity $v_{s}$, therefore one needs only to consider the $v_{s}$ dependent portions of $\mathcal{A}$. The relevant ground state energy is

$$
E_{\text {g.s. }}=\left(L_{x} L_{y} L_{z}\right) \rho v_{s}^{2} / 2,
$$

and the excitation free energy is given by the standard expression

$$
\mathcal{A}_{\text {excit }}=\left(k_{B} T\right) \sum_{\text {modes }} \log \left(1-e^{-\epsilon / k_{B} T}\right),
$$

where the excitation energies $\epsilon$ are those in the "rest frame", and are therefore Doppler shifted by the superflow:

$$
\epsilon=\hbar \omega(k)+v_{s} \hbar k_{x} .
$$

To second order in the superfluid velocity the free energy is given by

$$
\begin{aligned}
\mathcal{A}_{\text {excit }}- & \mathcal{A}_{\text {excit }}\left(v_{s}=0\right)=\left.\frac{v_{s}^{2}}{2} \frac{\partial^{2} \mathcal{A}_{\text {excit }}}{\partial v_{s}^{2}}\right|_{v_{s}=0} \\
= & -\frac{\hbar^{2} v_{s}^{2}}{2 k_{B} T} \sum_{\text {modes }} k_{x}^{2} \frac{e^{\hbar \omega(k) / k_{B} T}}{\left(e^{\hbar \omega(k) / k_{B} T}-1\right)^{2}} \\
= & -\left(L_{x} L_{y} L_{z}\right) \rho_{n} \frac{v_{s}^{2}}{2}
\end{aligned}
$$

where the last equality follows from the usual Landau derivation of the normal density 15.

The total change in Helmholtz free energy for variations in the superfluid velocity can be written as [17]

$$
\begin{aligned}
\Delta \mathcal{A} & =\left(L_{x} L_{y} L_{z}\right) \frac{\rho-\rho_{n}}{2} \Delta\left(v_{s}^{2}\right) \\
& =\left(L_{x} L_{y} L_{z}\right) \frac{\rho_{s}}{2} \Delta\left(v_{s}^{2}\right)=\left(L_{y} L_{z}\right) \rho_{s} v_{s} \frac{h}{m},
\end{aligned}
$$

Where the last equality corresponds to the change $\Delta v_{s}=h / m L_{x}$ due to the motion of the vortex across the ring.

By equating the work performed on the system (eq. 5) and the variation of free energy (eq. 11) the unknown coefficient $A$ in the general expression for the transverse force (4) can be determined:

$$
A=\rho_{s} \frac{h}{m} .
$$




\section{TOTAL TRANSVERSE FORCE AND CONCLUSIONS}

Having calculated the superfluid velocity part of the transverse force, there is the need to obtain one more component of it to completely determine the transverse force (4).

By considering TAN's result [2, 13], as written in eq. (3), it is clear that the coefficient of the vortex velocity $(A+B)$ is given by

$$
(A+B)=\rho_{s} \frac{h}{m},
$$

and this, along with the result for $A$ calculated in the previous section, yields unambiguously $B=0$, meaning that there is no transverse force depending on the normal fluid velocity. The total transverse force acting on a vortex would be simply written

$$
\boldsymbol{F}=\rho_{s} \frac{h}{m} \hat{\boldsymbol{z}} \times\left(\boldsymbol{v}_{V}-\boldsymbol{v}_{s}\right)
$$

with the transverse Iordanskii force vanishing exactly.

This is in general agreement with some direct calculations of the normal fluid velocity part of the transverse force based on the scattering of excitations by the vortex [1],18]. This calculations also show that the coefficient of the normal fluid velocity either vanishes or is much smaller than previously thought, in apparent conflict with Iordanskii's theory of the transverse force on a vortex [4, 12].

It is interesting to note that while this letter, in combination with TAN's result, yields an exactly vanishing Iordanskii force, the "direct" calculations mentioned above either give a non-zero result [4,12] or can only hint that it is small [11,13].

One must emphasize some differences in the assumptions about the asymptotic flow of excitations far away from the vortex. The authors calculating directly the Iordanskii force from the excitation scattering assume a homogeneous distribution of non-interacting phonons [4, 11, 12, 18, while the calculations used along this letter and references [2] and [13] include the effect of the vortex in the excitation distribution. This can explain some of the apparent discrepancies: a careful calculation of the Iordanskii force must include both the effects of the scattering of these excitations and the perturbation of the distribution functions by the vortex. Similar effects have been long known in the calculation of the viscous drag of a moving object in a fluid, namely the Stokes problem [19]. It may be possible to obtain independently the exact cancelation of the transverse Iordanskii force by including all the effects described in this paragraph. While this would be certainly desirable, it goes beyond the scope of this letter.

\section{ACKNOWLEDGMENTS}

I wish to thank David Thouless, Michael Geller, Jung Hoon Han, Greg Dash and John Rehr for numerous helpful discussions. This work was supported by the NSF grant DMR9528345 . 


\section{REFERENCES}

[1] R. B. Laughlin, Phys. Rev. B 23, 5632 (1981). B. I. Halperin, Phys. Rev. B 25, 2185 (1982).

[2] D. J. Thouless, P. Ao, and Q. Niu, Phys. Rev. Lett. 76, 3758-61 (1996).

[3] All forces herein referred are per unit vortex length.

[4] S. V. Iordanskii, Ann. Phys. (NY) 29, 335 (1964). S. V. Iordanskii, Sov. Phys. JETP 22, 160 (1966).

[5] L. Onsager, Nuovo Cimento Suppl. 6249 (1949); F. London, Superfluids II, J. Wiley, New York (1954).

[6] R. P. Feynman, Prog. Low Temp. Phys. I, North-Holland, chap. 1 (1955).

[7] Sir H. Lamb, Hydrodynamics, The University Press, Cambridge (1932), G. K. Batchelor An Introduction to Fluid Mechanics, Cambridge University Press (1967).

[8] C. F. Barenghi, R. J. Donnelly, and W. F. Vinen, J. Low Temp. Phys. 52, 189 (1983).

[9] R. J. Donnelly, Quantized Vortices in Helium II, Cambridge University Press, 1991.

[10] G. E. Volovik, JETP Lett. 62, 65 (1995).

[11] E. Demircan, P. Ao, and Q. Niu, Phys. Rev. B 52, 476 (1995).

[12] E. B. Sonin, Phys. Rev. B 55, 485 (1997).

[13] C. Wexler, and D. J. Thouless, cond-mat/9612059.

[14] M. Geller, C. Wexler, and D. J. Thouless, in preparation.

[15] L. Landau, J. Phys. V, 71 (1941).

[16] D. Chandler, An introduction to modern statistical mechanics, Oxford University Press, Oxford (1987).

[17] In fact, one can argue that this is the proper thermodynamic definition of the superfluid density: $\rho_{s} \equiv \frac{1}{V} \delta^{2} \mathcal{A} / \delta v_{s}^{2}$.

[18] C. Wexler, and D. J. Thouless, unpublished.

[19] E. H. Kennard, Kinetic Theory of Gases, McGraw-Hill, New York (1938). 


\section{FIGURES}

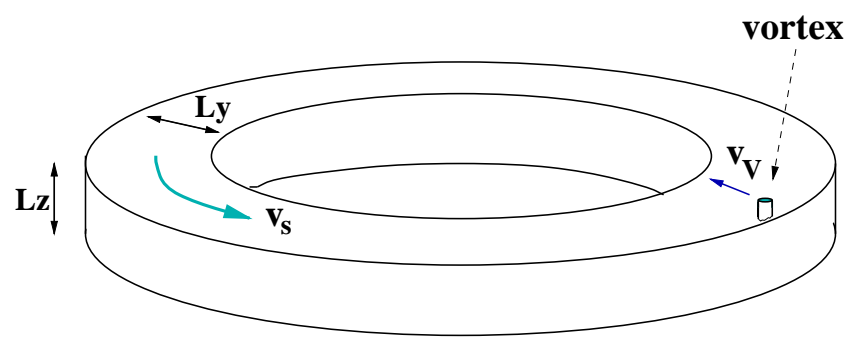

FIG. 1. Gedanken experiment: a vortex is adiabatically created at the outer edge of a toroid with circumference $L_{x}$, transported across the channel and annihilated at the inner edge thus increasing the total circulation around the toroid by one quantum of circulation $h / m$. 